\title{
Spatial coherence and density correlations of trapped Bose gases
}

\author{
M. Naraschewski \\ Jefferson Laboratory, Department of Physics, Harvard University, Cambridge MA 02138, USA \\ ITAMP, Harvard-Smithsonian Center for Astrophysics, 60 Garden Street, Cambridge, \\ MA 02138, USA \\ R. J. Glauber \\ Lyman Laboratory, Department of Physics, Harvard University, Cambridge MA 02138, USA
}

(June 26, 1998)

\begin{abstract}
We study first and second order coherence of trapped dilute Bose gases using appropriate correlation functions. Special attention is given to the discussion of second order or density correlations. Except for a small region around the surface of a Bose-Einstein condensate the correlations can be accurately described as those of a locally homogeneous gas with a spatially varying chemical potential. The degrees of first and second order coherence are therefore functions of temperature, chemical potential, and position. The second order correlation function is governed both by the tendency of bosonic atoms to cluster and by a strong repulsion at small distances due to atomic interactions. In present experiments both effects are of comparable magnitude. Below the critical temperature the range of the bosonic correlation is affected by the presence of collective quasi-particle excitations. The results of some recent experiments on second and third order coherence are discussed. It is shown that the relation between the measured quantities and the correlation functions is much weaker than previously assumed.
\end{abstract}

03.75.Fi,05.30.Jp

Typeset using REVTEX 


\section{INTRODUCTION}

The defining property of a Bose-Einstein condensate is the macroscopic occupation of a single one-particle quantum state [1], a condition often referred to a off-diagonal long range order. Even though Bose-Einstein condensation has been long known to exist in systems such as superfluid ${ }^{4} \mathrm{He}$, the off-diagonal long range order itself was inaccessible to direct experimental verification. The achievement of Bose-Einstein condensation in dilute alkali gases [2 [4], however, has revived interest in such direct experimental access. Indeed, after a number of theoretical studies [5-11], it has become possible to demonstrate off-diagonal long range order as made manifest by an interference experiment [12] and that finding has been confirmed by excellent agreement between experiment and theory [13,14.

The ability of a Bose-Einstein condensate to produce an interference pattern of high visibility is closely related to the first order coherence of an optical laser beam. More recently, two additional experiments have also explored certain aspects of the second [15] and third order coherence [16] of a trapped Bose condensed gas. It is therefore the aim of this paper to extend the theory of optical coherence [17] to the description of trapped dilute Bose gases. Our main focus will be on first and second order coherence.

The preferred tools to study coherence are correlation functions. While first order coherence relies on the properties of the reduced single-particle density matrix, which are expressed in the first order correlation function, second order coherence depends mainly on density correlations. Here, the strong short range repulsion between interacting atoms adds interesting new aspects to the discussion of atomic coherence, not present in its optical predecessor. Density correlations are an important object of study, of course, even apart from their bearing on coherence.

The paper is organized as follows. We introduce in Sec. II A appropriate first and second order correlation functions, which are then used in Sec. IIB to define coherence. It is the aim of the remainder of the paper to calculate these correlation functions for an externally trapped Bose gas. In most of our explicit calculations we assume a three-dimensional harmonic trapping potential and evaluate our results in the thermodynamic limit, i.e., we will not account for effects due to a finite atom number. This restriction is justified by the large number of atoms $\left(N \approx 10^{7}\right)$ in recent experiments. We thereby show that coherence is not only a function of temperature and chemical potential but also of the local position of the atoms. We consider first in Sec. III the case of an ideal Bose gas. This allows us to study some basic aspects of the problem and in particular the applicability of a local density approximation. Atomic interactions are added in Sec. IV. The main modifications are brought about by the existence of quasi-particle like excitations and by the strong short range repulsion between the atoms. In Sec. $\nabla$ we discuss the coherence properties that follow from the correlation functions calculated earlier. We also comment on the aforementioned

measurements of higher order coherence [15, 16] and a previous theoretical interpretation [18. We summarize our conclusions in Sec. VI.

\section{DEFINITIONS}




\section{A. Correlation functions}

We describe the atoms by a quantum field $\hat{\Psi}(\mathbf{r}, t)$ which obeys the equal-time commutation relations

$$
\begin{aligned}
{\left[\hat{\Psi}(\mathbf{r}, t), \hat{\Psi}^{\dagger}\left(\mathbf{r}^{\prime}, t\right)\right] } & =\delta\left(\mathbf{r}-\mathbf{r}^{\prime}\right) \\
{\left[\hat{\Psi}(\mathbf{r}, t), \hat{\Psi}\left(\mathbf{r}^{\prime}, t\right)\right] } & =0 .
\end{aligned}
$$

In general, physical properties of the gas can be expressed in terms of correlation functions, i.e., expectation values of the field operators of the type

$$
\left\langle\hat{\Psi}^{\dagger}\left(\mathbf{r}_{1}, t_{1}\right) \hat{\Psi}^{\dagger}\left(\mathbf{r}_{2}, t_{2}\right) \cdots \hat{\Psi}\left(\mathbf{r}_{2}^{\prime}, t_{2}^{\prime}\right) \hat{\Psi}\left(\mathbf{r}_{1}^{\prime}, t_{1}^{\prime}\right)\right\rangle .
$$

Here, a description of the dynamics using the Heisenberg picture has been tacitly assumed. However, we shall restrict ourselves to discussing spatial correlations at thermal equilibrium and at a fixed time. We therefore omit further mention of the time arguments. Temporal correlations have been discussed recently in the context of condensate phase fluctuations [10,11,19, 25].

For later convenience we introduce a special notation for the correlation functions

$$
\begin{aligned}
& G^{(1)}\left(\mathbf{r}, \mathbf{r}^{\prime}\right)=\left\langle\hat{\Psi}^{\dagger}(\mathbf{r}) \hat{\Psi}\left(\mathbf{r}^{\prime}\right)\right\rangle \\
& G^{(2)}\left(\mathbf{r}, \mathbf{r}^{\prime}\right)=\left\langle\hat{\Psi}^{\dagger}(\mathbf{r}) \hat{\Psi}^{\dagger}\left(\mathbf{r}^{\prime}\right) \hat{\Psi}\left(\mathbf{r}^{\prime}\right) \hat{\Psi}(\mathbf{r})\right\rangle
\end{aligned}
$$

The first order correlation function $G^{(1)}\left(\mathbf{r}, \mathbf{r}^{\prime}\right)$ contains the same information as the reduced one-particle density matrix which furnishes the expectation values of one-particle observables like the momentum or position density. The formal relation between first order correlation function and reduced one-particle density matrix is given by $G^{(1)}\left(\mathbf{r}, \mathbf{r}^{\prime}\right)=\rho^{(1)}\left(\mathbf{r}^{\prime}, \mathbf{r}\right)=$ $\left\langle\mathbf{r}^{\prime}\left|\rho^{(1)}\right| \mathbf{r}\right\rangle$. The first order correlation function can alternatively be expressed in terms of the Wigner function

$$
W(\mathbf{p}, \mathbf{q})=\frac{1}{(2 \pi \hbar)^{3}} \int d \mathbf{r} e^{-i \mathbf{p} \cdot \mathbf{r} / \hbar}\left\langle\hat{\Psi}^{\dagger}\left(\mathbf{q}-\frac{\mathbf{r}}{2}\right) \hat{\Psi}\left(\mathbf{q}+\frac{\mathbf{r}}{2}\right)\right\rangle
$$

which is a quantum mechanical analog of a classical single-particle phase space distribution. The connection between the quantum mechanical correlation function and statistical mechanics is indeed illustrated by the Fourier transform relation

$$
G^{(1)}\left(\mathbf{r}, \mathbf{r}^{\prime}\right)=\int d \mathbf{p} e^{-i \mathbf{p} \cdot\left(\mathbf{r}-\mathbf{r}^{\prime}\right) / \hbar} W\left(\mathbf{p}, \frac{\mathbf{r}+\mathbf{r}^{\prime}}{2}\right) .
$$

In contrast, the second order correlation function $G^{(2)}\left(\mathbf{r}, \mathbf{r}^{\prime}\right)$ expresses the joint probability of detecting two different atoms at the locations $\mathbf{r}$ and $\mathbf{r}^{\prime}$ respectively. Its connection with the density correlation function is given by

$$
\left\langle\hat{\Psi}^{\dagger}(\mathbf{r}) \hat{\Psi}(\mathbf{r}) \hat{\Psi}^{\dagger}\left(\mathbf{r}^{\prime}\right) \hat{\Psi}\left(\mathbf{r}^{\prime}\right)\right\rangle=G^{(2)}\left(\mathbf{r}, \mathbf{r}^{\prime}\right)+G^{(1)}(\mathbf{r}, \mathbf{r}) \delta\left(\mathbf{r}-\mathbf{r}^{\prime}\right) .
$$

It is because the function $G^{(2)}$ is defined as the average of a normally ordered product of field operators, that it expresses the correlation of distinct pairs of atoms. The delta function 
contribution in Eq. (2.8) represents by contrast the autocorrelation of individual atoms. Discussions of the coherence properties of fields are most conveniently phrased in terms of factorization of their correlation functions [17]. The autocorrelation contribution in Eq. (2.8) however stands in the way of any rigorous factorization of the density correlation into a product of functions of $\mathbf{r}$ and $\mathbf{r}^{\prime}$. We shall therefore concentrate on discussing the normally ordered correlation function $G^{(2)}\left(\mathbf{r}, \mathbf{r}^{\prime}\right)$ which can indeed meet the factorization condition under suitable physical circumstances.

\section{B. Coherence and Bose-Einstein condensation}

In the optical context coherence refers to a state of the system with interference properties that are as close as possible to those of a precisely defined classical field. In more technical terms, successive orders of coherence can be described by means of factorization conditions imposed upon a succession of field correlation functions. The analog of $n$-th order coherence for the atomic field would require all normally ordered correlation functions of order $m \leq n$ to factorize into a form

$$
\left\langle\hat{\Psi}^{\dagger}\left(\mathbf{r}_{1}\right) \hat{\Psi}^{\dagger}\left(\mathbf{r}_{2}\right) \cdots \hat{\Psi}\left(\mathbf{r}_{2}^{\prime}\right) \hat{\Psi}\left(\mathbf{r}_{1}^{\prime}\right)\right\rangle=\psi^{*}\left(\mathbf{r}_{1}\right) \psi^{*}\left(\mathbf{r}_{2}\right) \cdots \psi\left(\mathbf{r}_{2}^{\prime}\right) \psi\left(\mathbf{r}_{1}^{\prime}\right)
$$

i.e., into products of the same complex valued function $\psi(\mathbf{r})$. If the field were in a pure coherent state, the factors $\psi(\mathbf{r})$ could be regarded as field-expectation values $\langle\hat{\Psi}(\mathbf{r})\rangle$. This identification, however, is by no means necessary to secure the factorizations of Eq. (2.9). It would even contradict the strict conservation of the total atom number.

To identify the function $\psi(\mathbf{r})$ we begin by considering first order coherence, which secures the maximum effects of intensity interference between spatially separated parts of the system. In this sense, perfect first order coherence

$$
G^{(1)}\left(\mathbf{r}, \mathbf{r}^{\prime}\right)=\psi^{*}(\mathbf{r}) \psi\left(\mathbf{r}^{\prime}\right)
$$

with

$$
\psi(\mathbf{r})=\sqrt{N}\langle\mathbf{r} \mid \phi\rangle
$$

will hold if all atoms occupy the same one-particle state $|\phi\rangle$. Here $N$ denotes the total atom number of the system. This definition is an idealization of the Penrose and Onsager definition of Bose-Einstein condensation [1] which requires the reduced one-particle density matrix to have a single macroscopic eigenvalue. The function $\psi(\mathbf{r})$ can thus be introduced without breaking the gauge symmetry associated with the conservation of total atom number. The modulus of $\psi(\mathbf{r})$ can be reexpressed in terms of the first order correlation function as

$$
|\psi(\mathbf{r})|=\sqrt{G^{(1)}(\mathbf{r}, \mathbf{r})} .
$$

In addition to the factorization of $G^{(1)}$, perfect second order coherence implies the factorization condition

$$
G^{(2)}\left(\mathbf{r}, \mathbf{r}^{\prime}\right)=|\psi(\mathbf{r})|^{2}\left|\psi\left(\mathbf{r}^{\prime}\right)\right|^{2}
$$


so that

$$
G^{(2)}\left(\mathbf{r}, \mathbf{r}^{\prime}\right)=G^{(1)}(\mathbf{r}, \mathbf{r}) G^{(1)}\left(\mathbf{r}^{\prime}, \mathbf{r}^{\prime}\right)
$$

First order coherence does not, of course, require second order coherence to hold. It does, however, imply a weaker factorization property of the function $G^{(2)}$ [26] which includes the statement

$$
G^{(2)}\left(\mathbf{r}, \mathbf{r}^{\prime}\right)=g_{2}|\psi(\mathbf{r})|^{2}\left|\psi\left(\mathbf{r}^{\prime}\right)\right|^{2}
$$

where $g_{2}$ is a non-negative real number. For example, a first order coherent system with definite total atom number can achieve second order coherence, i.e. $g_{2}=1$, only to an accuracy of order $O(1 / N)$.

In fact, any real atomic system can exhibit coherence only in an approximate way. In order to define a local measure of coherence we introduce correlation functions which are normalized to attain unit modulus in the case of perfect coherence. The degree of first order coherence, in this sense, is expressed by

$$
g^{(1)}\left(\mathbf{r}, \mathbf{r}^{\prime}\right)=\frac{G^{(1)}\left(\mathbf{r}, \mathbf{r}^{\prime}\right)}{\sqrt{G^{(1)}(\mathbf{r}, \mathbf{r})} \sqrt{G^{(1)}\left(\mathbf{r}^{\prime}, \mathbf{r}^{\prime}\right)}}
$$

while

$$
g^{(2)}\left(\mathbf{r}, \mathbf{r}^{\prime}\right)=\frac{G^{(2)}\left(\mathbf{r}, \mathbf{r}^{\prime}\right)}{G^{(1)}(\mathbf{r}, \mathbf{r}) G^{(1)}\left(\mathbf{r}^{\prime}, \mathbf{r}^{\prime}\right)}
$$

is a certain measure of second order coherence. In the language of a classical field theory $g^{(1)}$ characterizes local fluctuations of the phase of the complex field amplitude, while $g^{(2)}$ is related to fluctuations of its modulus. The most important property of $g^{(1)}$ is its relation to the contrast achievable in an interference experiment [27]. The function $g^{(2)}$, on the other hand, expresses the tendency of atoms either to cluster or to remain spatially separated.

In many experimental contexts, it will be difficult, to collect enough data to determine the dependence of the correlation functions on the two coordinates $\mathbf{r}$ and $\mathbf{r}^{\prime}$. It may then be simpler to measure volume integrated correlation functions

$$
\begin{aligned}
& G^{(1)}(\mathbf{r})=\int d \mathbf{R} G^{(1)}\left(\mathbf{R}-\frac{\mathbf{r}}{2}, \mathbf{R}+\frac{\mathbf{r}}{2}\right) \\
& G^{(2)}(\mathbf{r})=\int d \mathbf{R} G^{(2)}\left(\mathbf{R}-\frac{\mathbf{r}}{2}, \mathbf{R}+\frac{\mathbf{r}}{2}\right),
\end{aligned}
$$

in which a spatial integration over the mean coordinate has been performed. In condensed matter physics, for example, scattering experiments are often used to obtain information about the volume integrated second order correlation function. We define normalized versions of these functions

$$
\begin{aligned}
g^{(1)}(\mathbf{r}) & =\frac{\int d \mathbf{R} G^{(1)}\left(\mathbf{R}-\frac{\mathbf{r}}{2}, \mathbf{R}+\frac{\mathbf{r}}{2}\right)}{\int d \mathbf{R} \sqrt{G^{(1)}\left(\mathbf{R}-\frac{\mathbf{r}}{2}, \mathbf{R}-\frac{\mathbf{r}}{2}\right)} \sqrt{G^{(1)}\left(\mathbf{R}+\frac{\mathbf{r}}{2}, \mathbf{R}+\frac{\mathbf{r}}{2}\right)}} \\
g^{(2)}(\mathbf{r}) & =\frac{\int d \mathbf{R} G^{(2)}\left(\mathbf{R}-\frac{\mathbf{r}}{2}, \mathbf{R}+\frac{\mathbf{r}}{2}\right)}{\int d \mathbf{R} G^{(1)}\left(\mathbf{R}-\frac{\mathbf{r}}{2}, \mathbf{R}-\frac{\mathbf{r}}{2}\right) G^{(1)}\left(\mathbf{R}+\frac{\mathbf{r}}{2}, \mathbf{R}+\frac{\mathbf{r}}{2}\right)},
\end{aligned}
$$


which can also be interpreted as weighted volume averages of the normalized correlation functions of Eqs. (2.16) and (2.17).

It will be the aim of the following sections to calculate the correlation functions we have defined for trapped dilute Bose gases at finite temperatures. The main emphasis will be put on systems that are large enough to allow a local density approximation.

\section{IDEAL GASES}

The basic concepts for calculating the correlation functions of a trapped gas are most easily described for ideal gases with temperatures above the critical temperature. The presence of a Bose-Einstein condensate at temperatures below the critical temperature gives rise to additional features which will be discussed in Sec. [IIB. The influence of atomic interactions on both uncondensed and condensed gases will be later dealt with in Sec. IV]

\section{A. Temperatures above $T_{c}$}

In the simplest case, we consider an ideal Bose gas at temperature $T$ trapped by an external potential $V(\mathbf{r})$. In the grand canonical ensemble different one-particle energy eigenstates $u_{\mathbf{n}}(\mathbf{r})$ of the trapping potential are populated independently. As a result we can write

$$
\begin{aligned}
G^{(1)}\left(\mathbf{r}, \mathbf{r}^{\prime}\right)= & \sum_{\mathbf{m}} u_{\mathbf{m}}^{*}(\mathbf{r}) u_{\mathbf{m}}\left(\mathbf{r}^{\prime}\right)\left\langle\hat{a}_{\mathbf{m}}^{\dagger} a_{\mathbf{m}}\right\rangle \\
G^{(2)}\left(\mathbf{r}, \mathbf{r}^{\prime}\right)= & \sum_{\mathbf{k l m} \mathbf{n}} u_{\mathbf{k}}^{*}(\mathbf{r}) u_{\mathbf{l}}^{*}\left(\mathbf{r}^{\prime}\right) u_{\mathbf{m}}\left(\mathbf{r}^{\prime}\right) u_{\mathbf{n}}(\mathbf{r})\left\langle\hat{a}_{\mathbf{k}}^{\dagger} \hat{a}_{\mathbf{l}}^{\dagger} \hat{a}_{\mathbf{m}} \hat{a}_{\mathbf{n}}\right\rangle \\
= & \left\langle\hat{\Psi}^{\dagger}(\mathbf{r}) \hat{\Psi}(\mathbf{r})\right\rangle\left\langle\hat{\Psi}^{\dagger}\left(\mathbf{r}^{\prime}\right) \hat{\Psi}\left(\mathbf{r}^{\prime}\right)\right\rangle+\left|\left\langle\hat{\Psi}^{\dagger}\left(\mathbf{r}^{\prime}\right) \hat{\Psi}(\mathbf{r})\right\rangle\right|^{2} \\
& +\sum_{\mathbf{m}}\left|u_{\mathbf{m}}(\mathbf{r})\right|^{2}\left|u_{\mathbf{m}}\left(\mathbf{r}^{\prime}\right)\right|^{2}\left(\left\langle\hat{a}_{\mathbf{m}}^{\dagger} \hat{a}_{\mathbf{m}}^{\dagger} \hat{a}_{\mathbf{m}} \hat{a}_{\mathbf{m}}\right\rangle-2\left\langle\hat{a}_{\mathbf{m}}^{\dagger} \hat{a}_{\mathbf{m}}\right\rangle\left\langle\hat{a}_{\mathbf{m}}^{\dagger} \hat{a}_{\mathbf{m}}\right\rangle\right) .
\end{aligned}
$$

The statistical independence we have assumed for the mode occupations would not, on the other hand, hold precisely for a canonical ensemble, i.e., a system with a fixed total atom number. However, the error in the correlation function induced thereby is only of the order $O(1 / N)$ and can be neglected for large systems.

The occupation fluctuations characteristic of the grand canonical ensemble make the last line of Eq. (3.2) vanish. The second order correlation functions can then be written as

$$
\begin{aligned}
G^{(2)}\left(\mathbf{r}, \mathbf{r}^{\prime}\right) & =G^{(1)}(\mathbf{r}, \mathbf{r}) G^{(1)}\left(\mathbf{r}^{\prime}, \mathbf{r}^{\prime}\right)+\left|G^{(1)}\left(\mathbf{r}, \mathbf{r}^{\prime}\right)\right|^{2} \\
g^{(2)}\left(\mathbf{r}, \mathbf{r}^{\prime}\right) & =1+\left|g^{(1)}\left(\mathbf{r}, \mathbf{r}^{\prime}\right)\right|^{2}
\end{aligned}
$$

The second term of $G^{(2)}$ or $g^{(2)}$ is a quantum statistical exchange term. It manifests a tendency of bosonic atoms to cluster together. In the case of fermions this term would have a negative sign reflecting the influence of the Pauli exclusion principle.

Since we may freely use the grand canonical ensemble at temperatures above $T_{c}$, we need only determine the first order correlation functions $G^{(1)}\left(\mathbf{r}, \mathbf{r}^{\prime}\right)$ and $g^{(1)}\left(\mathbf{r}, \mathbf{r}^{\prime}\right)$. Furthermore, no condensate exists in this regime and the normalized correlation functions have the limiting values 


$$
\begin{array}{cc}
g^{(1)}(\mathbf{r}, \mathbf{r})=1 & \lim _{\left|\mathbf{r}-\mathbf{r}^{\prime}\right| \rightarrow \infty} g^{(1)}\left(\mathbf{r}, \mathbf{r}^{\prime}\right)=0 \\
g^{(2)}(\mathbf{r}, \mathbf{r})=2 & \lim _{\left|\mathbf{r}-\mathbf{r}^{\prime}\right| \rightarrow \infty} g^{(2)}\left(\mathbf{r}, \mathbf{r}^{\prime}\right)=1 .
\end{array}
$$

In the following we compute the length scale of $\mathbf{r}-\mathbf{r}^{\prime}$ over which the correlation functions decrease.

\section{Three-dimensional harmonic oscillator potential}

As a first step we calculate the first order correlation function for the case of a spherical harmonic oscillator potential $V(\mathbf{r})=m \omega^{2} r^{2} / 2$ with angular trap frequency $\omega$. We therefore occupy the harmonic oscillator states in Eq. (3.1) with a Bose-Einstein distribution, so that

$$
G^{(1)}\left(\mathbf{r}, \mathbf{r}^{\prime}\right)=\sum_{\mathbf{m}} u_{\mathbf{m}}^{*}(\mathbf{r}) u_{\mathbf{m}}\left(\mathbf{r}^{\prime}\right) \frac{z e^{-\beta \tilde{\epsilon}_{\mathbf{m}}}}{1-z e^{-\beta \tilde{\epsilon}_{\mathbf{m}}}}
$$

with the inverse temperature $\beta=1 / k_{B} T$, the fugacity $z=e^{\beta \mu}$, and the chemical potential $\mu$. The energies $\tilde{\epsilon}_{\mathbf{m}}$ have the zero point energy, $3 \hbar \omega / 2$, removed in order to limit the fugacity to values between zero and one, as in a homogeneous gas. If the summand of Eq. (3.7) is expanded in powers of $z$, the remaining sum over the state indices $\mathbf{m}$ can be compactly expressed in terms of the single-particle propagator $G_{0}\left(\mathbf{r}^{\prime}, \mathbf{r}, t\right)=\left\langle\mathbf{r}^{\prime}|\exp (-i H t)| \mathbf{r}\right\rangle$ for motion governed by the harmonic oscillator Hamiltonian, $H$. We obtain

$$
\begin{aligned}
G^{(1)}\left(\mathbf{r}, \mathbf{r}^{\prime}\right) & =\sum_{\mathbf{m}} u_{\mathbf{m}}^{*}(\mathbf{r}) u_{\mathbf{m}}\left(\mathbf{r}^{\prime}\right) z e^{-\beta \tilde{\epsilon}_{\mathbf{n}}} \sum_{k=0}^{\infty} z^{k} e^{-k \beta \tilde{\epsilon}_{\mathbf{n}}} \\
& =\sum_{k=1}^{\infty} z^{k} \exp (3 k \beta \hbar \omega / 2) G_{0}\left(\mathbf{r}^{\prime}, \mathbf{r}, t=-i \hbar k \beta\right) \\
& =\sum_{k=1}^{\infty} z^{k} \tilde{G}_{0}\left(\mathbf{r}^{\prime}, \mathbf{r}, k \beta \hbar \omega\right)
\end{aligned}
$$

where

$$
\tilde{G}_{0}\left(\mathbf{r}^{\prime}, \mathbf{r}, \tau\right)=\left(\frac{m \omega}{\pi \hbar\left(1-e^{-2 \tau}\right)}\right)^{3 / 2} \exp \left[-\frac{m \omega}{\hbar} \frac{\left(r^{2}+r^{\prime 2}\right)(\cosh \tau-1)+\left(\mathbf{r}-\mathbf{r}^{\prime}\right)^{2}}{2 \sinh \tau}\right]
$$

is derived from the Green's function for the three-dimensional harmonic oscillator [28]. The fugacity $z$ must be determined self-consistently by using the relation 29.

$$
N=\sum_{k=1}^{\infty} \frac{z^{k}}{\left(1-e^{-k \beta \hbar \omega}\right)^{3}}
$$

in order to insure the proper total atom number $N$.

A considerable simplification can be achieved if the energy spacing of the trap levels is much smaller than the thermal energy, i.e., $\beta \hbar \omega \ll 1$. In that case $\tilde{G}_{0}$ reduces to

$$
\tilde{G}_{0}\left(\mathbf{r}^{\prime}, \mathbf{r}, \tau\right)=\left(\frac{m \omega}{2 \pi \hbar \tau}\right)^{3 / 2} \exp \left[-\frac{m \omega}{\hbar} \frac{\left(r^{2}+r^{\prime 2}\right) \tau / 2+\left(\mathbf{r}-\mathbf{r}^{\prime}\right)^{2} / \tau}{2}\right]
$$


and the sum for $G^{(1)}$ can be written in the form

$$
\begin{aligned}
G^{(1)}\left(\mathbf{r}, \mathbf{r}^{\prime}\right) & =\frac{1}{\lambda_{T}^{3}} \sum_{k=1}^{\infty} \frac{z^{k}}{k^{3 / 2}} \exp \left[-\frac{\left[V(\mathbf{r})+V\left(\mathbf{r}^{\prime}\right)\right] / 2}{k_{B} T}\right]^{k} \exp \left[-\pi \frac{\left(\mathbf{r}-\mathbf{r}^{\prime}\right)^{2}}{\lambda_{T}^{2}}\right]^{1 / k} \\
& =\frac{1}{\lambda_{T}^{3}} g_{3 / 2}\left(\exp \left[\frac{\mu-\left[V(\mathbf{r})+V\left(\mathbf{r}^{\prime}\right)\right] / 2}{k_{B} T}\right], \exp \left[-\pi \frac{\left(\mathbf{r}-\mathbf{r}^{\prime}\right)^{2}}{\lambda_{T}^{2}}\right]\right)
\end{aligned}
$$

where we have used the thermal wavelength

$$
\lambda_{T}=\hbar \sqrt{2 \pi / m k_{B} T}
$$

In addition we have introduced the generalized Bose function

$$
g_{\alpha}(x, y)=\sum_{k=1}^{\infty} \frac{x^{k} y^{1 / k}}{k^{\alpha}}
$$

which is related to the commonly used Bose function $g_{\alpha}(x)=\sum_{k=1}^{\infty} x^{k} / k^{\alpha}$ and to the Riemann zeta function $\zeta(\alpha)=\sum_{k=1}^{\infty} 1 / k^{\alpha}$ through

$$
g_{\alpha}(x)=g_{\alpha}(x, 1), \quad \zeta(\alpha)=g_{\alpha}(1)=g_{\alpha}(1,1) .
$$

\section{Local density approximation}

The implications of Eq. (3.12) can be made clearer by deriving an approximate form for it from Eq. (2.7). There $G^{(1)}$ was expressed in terms of the Wigner function. We now employ the local density approximation which assumes the Wigner function, $W(\mathbf{p}, \mathbf{q})$, to be locally identical to the momentum distribution of a spatially homogeneous system with constant potential energy equal to the local value $V(\mathbf{q})$

$$
W(\mathbf{p}, \mathbf{q})=\frac{1}{(2 \pi \hbar)^{3}} \frac{1}{\exp \left[\left(p^{2} / 2 m+V(\mathbf{q})-\mu\right) / k_{B} T\right]-1} .
$$

This approximation implies that the atoms see an effective chemical potential $\mu(\mathbf{q})=\mu-$ $V(\mathbf{q})$ which varies with position. If we Fourier transform this Wigner function as required by Eq. (2.7) the correlation function can be expressed as

$$
G^{(1)}\left(\mathbf{r}, \mathbf{r}^{\prime}\right)=\frac{1}{\lambda_{T}^{3}} g_{3 / 2}\left(\exp \left[\frac{\mu-V\left[\left(\mathbf{r}+\mathbf{r}^{\prime}\right) / 2\right]}{k_{B} T}\right], \exp \left[-\pi \frac{\left(\mathbf{r}-\mathbf{r}^{\prime}\right)^{2}}{\lambda_{T}^{2}}\right]\right) .
$$

The global chemical potential $\mu$ must then be chosen to secure the correct total atom number

$$
N=\int d \mathbf{r} G^{(1)}(\mathbf{r}, \mathbf{r})
$$

We can now compare Eq. (3.17) with Eq. (3.12). These expressions for $G^{(1)}$ in fact coincide for equal arguments $\mathbf{r}=\mathbf{r}^{\prime}$. However, for $\mathbf{r} \neq \mathbf{r}^{\prime}$ they treat the potential energy in a slightly different way. The accuracy of the local density approximation evidently requires 
that the external potential vary slowly on a length scale given by $\lambda_{T}$. For a relative separation $\mathbf{r}-\mathbf{r}^{\prime}$ of this magnitude, the difference of the potential energy between Eqs. (3.12) and (3.17) is only of the order $(\hbar \omega)^{2} / k_{B} T$ and can thus be neglected. This result can be no surprise if we consider that the thermal wavelength, written as $\lambda_{T}=\sqrt{2 \pi \hbar \omega / k_{B} T} a_{0}$, becomes smaller than the natural length unit $a_{0}=\sqrt{\hbar / m \omega}$ of the trapping potential precisely in the limit $\hbar \omega \ll k_{B} T$. For the trap parameters of Ref. [12], the thermal wavelength assumes a value of $\lambda_{T}=0.4 \mu \mathrm{m}$ at the critical temperature, which is three to ten times smaller than the harmonic oscillator length units of the anisotropic trapping potential.

We have thus shown that both the density distribution and correlations implicit in $G^{(1)}$ are well described by the local density approximation, as long as the separation of the trap levels is small compared to the thermal energy. Of course, application of the local density approximation is not restricted to an isotropic harmonic oscillator potential. It can be applied to any trapping potential $V(\mathbf{r})$ as long as the thermal wavelength is small compared to the length scale of the spatial variations of the potential. Since the correlation function decreases on the scale of the thermal wavelength it is consistent to use for small relative separations $\left(r<a_{0}\right)$ the alternative definitions

$$
\begin{aligned}
g^{(1)}(\mathbf{r}) & =\frac{\int d \mathbf{R} G^{(1)}\left(\mathbf{R}-\frac{\mathbf{r}}{2}, \mathbf{R}+\frac{\mathbf{r}}{2}\right)}{\int d \mathbf{R} G^{(1)}(\mathbf{R}, \mathbf{R})} \\
g^{(2)}(\mathbf{r}) & =\frac{\int d \mathbf{R} G^{(2)}\left(\mathbf{R}-\frac{\mathbf{r}}{2}, \mathbf{R}+\frac{\mathbf{r}}{2}\right)}{\int d \mathbf{R}\left|G^{(1)}(\mathbf{R}, \mathbf{R})\right|^{2}}
\end{aligned}
$$

for the normalization of the volume integrated correlation functions.

For a harmonic oscillator potential the integration over the mean coordinate $\mathbf{R}=(\mathbf{r}+$ $\left.\mathbf{r}^{\prime}\right) / 2$ of Eq. (3.17) can easily be carried out [30] by using the series expansion of $g_{3 / 2}$. The results for the volume integrated correlation functions of Eqs. (2.18) and (3.19) are then

$$
\begin{aligned}
G^{(1)}(\mathbf{r}) & =\left(\frac{k_{B} T}{\hbar \omega}\right)^{3} g_{3}\left(\exp \left[\frac{\mu}{k_{B} T}\right], \exp \left[-\pi \frac{r^{2}}{\lambda_{T}^{2}}\right]\right) \\
g^{(1)}(\mathbf{r}) & =\frac{g_{3}\left(\exp \left[\frac{\mu}{k_{B} T}\right], \exp \left[-\pi \frac{r^{2}}{\lambda_{T}^{2}}\right]\right)}{g_{3}\left(\exp \left[\frac{\mu}{k_{B} T}\right]\right)} .
\end{aligned}
$$

The latter formula applies equally well to the case of an anisotropic harmonic potential when the replacement $\omega=\left(\omega_{x} \omega_{y} \omega_{z}\right)^{1 / 3}$ is made. As in the well known case of a spatially homogeneous system, the correlations decay on a length scale given by the thermal wavelength $\lambda_{T}$. However, the Bose function $g_{3}$ appropriate to a harmonic oscillator potential is closer to a linear function of its argument than the function $g_{3 / 2}$ characteristic of a homogeneous system. The properties of a Bose-Einstein distribution are thus less visible in the volume integrated correlation function of a harmonically trapped system than in that of a homogeneous system, since the atoms away from the trap center experience a lower local chemical potential. In view of this reduced tendency towards condensation for larger radii it may be an acceptable approximation to assume a Maxwell-Boltzmann occupation distribution of the trap levels, or equivalently to restrict the summation in Eq. (3.12) to its lowest order term. In this approximation we obtain the simple expressions 


$$
g^{(1)}(\mathbf{r})=\exp \left(-\pi \frac{r^{2}}{\lambda_{T}^{2}}\right), \quad g^{(2)}(\mathbf{r})=1+\exp \left(-2 \pi \frac{r^{2}}{\lambda_{T}^{2}}\right) .
$$

Numerical calculations confirm (cf. Fig. 1) the accuracy of these simple expressions even at temperatures very close to the critical temperature.

\section{B. Temperatures below $T_{c}$}

In Sec. IIIA we have calculated the first and second order correlation functions for uncondensed systems. The presence of a Bose-Einstein condensate, however, will lead to significant changes, especially in the second order correlation function.

In principle, the presence of a Bose-Einstein condensate has no effect on the previously discussed expression, Eq. (3.1), for the first order correlation function $G^{(1)}$. However, due to the relatively long ranged coherence of the condensate we have to perform the local density approximation in a slightly different way. We therefore introduce a Wigner function, $W_{T}(\mathbf{p}, \mathbf{q})$, which describes only the uncondensed atoms. The condensate, which corresponds to the ground state of the bare external potential, is still considered to have a wave function $\psi(\mathbf{r})$ that obeys the Schrödinger equation

$$
\left(-\frac{\hbar^{2} \nabla^{2}}{2 m}+V(\mathbf{r})-\mu\right) \psi(\mathbf{r})=0
$$

with $\mu=\hbar\left(\omega_{x}+\omega_{y}+\omega_{z}\right) / 2$ corresponding to the zero point energy of the oscillator potential. The first order correlation function can then be decomposed into a condensed and an uncondensed or thermal part

$$
G^{(1)}\left(\mathbf{r}, \mathbf{r}^{\prime}\right)=\psi^{*}(\mathbf{r}) \psi\left(\mathbf{r}^{\prime}\right)+G_{T}^{(1)}\left(\mathbf{r}, \mathbf{r}^{\prime}\right)
$$

with

$$
G_{T}^{(1)}\left(\mathbf{r}, \mathbf{r}^{\prime}\right)=\int d \mathbf{p} e^{-i \mathbf{p} \cdot\left(\mathbf{r}-\mathbf{r}^{\prime}\right) / \hbar} W_{T}\left(\mathbf{p}, \frac{\mathbf{r}+\mathbf{r}^{\prime}}{2}\right) .
$$

Again, the thermal Wigner function $W_{T}$ describes the uncondensed part of the full Wigner function. The thermal correlation function $G_{T}^{(1)}$ is given by Eq. (3.17), since the momentum integration which led to Eq. (3.17) does not account for the existence of a condensate. However, in contrast to Eq. (3.24), the chemical potential in $W_{T}$ is set equal to zero, $(\mu=0)$, as it must be in the local density approximation. The occupation of the condensate mode, furthermore, has to be determined from the normalization condition

$$
N=\int d \mathbf{r}|\psi(\mathbf{r})|^{2}+\int d \mathbf{r} G_{T}^{(1)}(\mathbf{r}, \mathbf{r}) .
$$

The limiting values of the normalized first order correlation function

$$
\begin{aligned}
g^{(1)}(\mathbf{r}, \mathbf{r}) & =1 \\
\lim _{\left|\mathbf{r}-\mathbf{r}^{\prime}\right| \rightarrow \infty} g^{(1)}\left(\mathbf{r}, \mathbf{r}^{\prime}\right) & =\frac{\psi^{*}(\mathbf{r}) \psi\left(\mathbf{r}^{\prime}\right)}{\sqrt{G^{(1)}(\mathbf{r}, \mathbf{r})} \sqrt{G^{(1)}\left(\mathbf{r}^{\prime}, \mathbf{r}^{\prime}\right)}}
\end{aligned}
$$


differ from those of an uncondensed gas (cf. Eq. (3.5)) by the possibility of off-diagonal long range order. The existence of a condensate causes the correlation length of $g^{(1)}$ to be much larger than the thermal wavelength $\lambda_{T}$. In the case of a spatially homogeneous gas the correlation extends throughout the entire system. The degree of first order coherence for large separations is then just given by the local condensate fraction.

The second order correlation function is affected by Bose-Einstein condensation in a more interesting way, as can be seen in Eq. (3.2). The fluctuations of the number of atoms in the condensate mode depend sensitively on the statistical ensemble that is assumed for the calculation. The fluctuations characteristic of the grand canonical ensemble greatly overestimate the condensate fluctuations in any experiment with a fixed total atom number. Instead we assume canonical condensate fluctuations which are of the order [31]

$$
\left\langle\hat{a}_{0}^{\dagger} \hat{a}_{0}^{\dagger} \hat{a}_{0} \hat{a}_{0}\right\rangle=\left\langle\hat{a}_{0}^{\dagger} \hat{a}_{0}\right\rangle\left\langle\hat{a}_{0}^{\dagger} \hat{a}_{0}\right\rangle+O(N) .
$$

In the limit of a large system we can neglect the last term since it is much smaller than the first term which is of the order $O\left(N^{2}\right)$. To calculate the second order correlation function we refer back to Eq. (3.2) and note that the fluctuations of the uncondensed modes can be well approximated by using the grand canonical ensemble. For the condensate mode, on the other hand, we can write $\psi(\mathbf{r})=\sqrt{\left\langle\hat{a}_{0}^{\dagger} \hat{a}_{0}\right\rangle} u_{0}(\mathbf{r})$, so that we have

$$
\begin{aligned}
G^{(2)}\left(\mathbf{r}, \mathbf{r}^{\prime}\right) & =G^{(1)}(\mathbf{r}, \mathbf{r}) G^{(1)}\left(\mathbf{r}^{\prime}, \mathbf{r}^{\prime}\right)+\left|G^{(1)}\left(\mathbf{r}, \mathbf{r}^{\prime}\right)\right|^{2}-|\psi(\mathbf{r})|^{2}\left|\psi\left(\mathbf{r}^{\prime}\right)\right|^{2} \\
g^{(2)}\left(\mathbf{r}, \mathbf{r}^{\prime}\right) & =1+\left|g^{(1)}\left(\mathbf{r}, \mathbf{r}^{\prime}\right)\right|^{2}-\frac{|\psi(\mathbf{r})|^{2}\left|\psi\left(\mathbf{r}^{\prime}\right)\right|^{2}}{G^{(1)}(\mathbf{r}, \mathbf{r}) G^{(1)}\left(\mathbf{r}^{\prime}, \mathbf{r}^{\prime}\right)}
\end{aligned}
$$

The limit for large relative separations is again given by

$$
\lim _{\left|\mathbf{r}-\mathbf{r}^{\prime}\right| \rightarrow \infty} g^{(2)}\left(\mathbf{r}, \mathbf{r}^{\prime}\right)=1
$$

A certain measure [18] of second order coherence is

$$
g^{(2)}(\mathbf{r}, \mathbf{r})=2-\left(\frac{|\psi(\mathbf{r})|^{2}}{G^{(1)}(\mathbf{r}, \mathbf{r})}\right)^{2} .
$$

This function, which is determined by the ratio of the local condensate density to the total density must reduce to unity everywhere in order to describe perfect second order coherence. In general, it can only be close to one near the center of the trap, where the condensate density is large. The coherence properties of the thermal cloud outside the condensate, i.e. where $\psi(\mathbf{r})=0$, are not greatly different from those of an uncondensed gas.

\section{STRONGLY INTERACTING, DILUTE GASES}

In Sec. III we have studied the basic properties of the first and second order correlation functions of a trapped ideal gas. In this section we discuss the way in which atomic interactions modify these results. In order to deal with recent Bose condensation experiments [2 [4] we focus on gases which are very dilute, but which contain atoms with a strong mutual repulsion. In this section we discuss only Bose condensed gases. The properties of an uncondensed gas can easily be obtained as a specialisation of the following treatment. 


\section{A. First order correlation function}

In the presence of atomic interactions, the condensate wave function obeys the finite temperature Gross-Pitaevskii equation [30] (cf. Eq. (3.24))

$$
\left(-\frac{\hbar^{2} \nabla^{2}}{2 m}+V(\mathbf{r})+U\left[|\psi(\mathbf{r})|^{2}+2 n_{T}(\mathbf{r})\right]-\mu\right) \psi(\mathbf{r})=0
$$

where the atomic interaction strength is measured by $U=4 \pi \hbar^{2} a / m$, with $a$ denoting the two-body scattering length. The local density of thermal atoms $n_{T}(\mathbf{r})$ is given by the stillto-be determined thermal part of the first order correlation function (cf. Eqs. (3.25) and $(3.26))$,

$$
n_{T}(\mathbf{r})=G_{T}^{(1)}(\mathbf{r}, \mathbf{r})=\int d \mathbf{p} W_{T}(\mathbf{p}, \mathbf{r})
$$

In the case of an interacting gas, the local density approximation has to be applied not only to the distribution of thermal atoms but also to the condensate wave function in order to be consistent. Otherwise, the local kinetic energy of the condensate wave function could be larger than the kinetic energy of the low lying elementary excitations. Details of this argument will be presented later. We have therefore to neglect the kinetic energy term in the Gross-Pitaevskii equation (4.1), and that is appropriate if the kinetic energy is much smaller than the interaction energy. Under present experimental conditions 30] this condition is well fullfilled in the core of the condensate. However, the local density approximation can not be applied in a small region around the surface of the condensate, where the interaction energy ceases to dominate the kinetic energy. The radial extent of this region is usually called the healing length. It has a fixed thickness and therefore a vanishing relevance in the thermodynamic limit. This local density or Thomas-Fermi approximation has already been used frequently for the description of Bose-Einstein condensation experiments. In this limit, the condensate wave function is given by

$$
\psi(\mathbf{r})=\sqrt{\frac{\mu-V(\mathbf{r})-2 U n_{T}(\mathbf{r})}{U}}
$$

for positive arguments of the square root and by zero otherwise [32].

Since the local density approximation fails close to the surface of the condensate we are only able to study correlation functions with both of their spatial arguments located within the condensate or outside of it. Inside the condensate, the first order correlation function is given by

$$
G^{(1)}\left(\mathbf{r}, \mathbf{r}^{\prime}\right)=\psi^{*}(\mathbf{r}) \psi\left(\mathbf{r}^{\prime}\right)+\int d \mathbf{p} e^{-i \mathbf{p} \cdot\left(\mathbf{r}-\mathbf{r}^{\prime}\right) / \hbar} W_{T}\left(\mathbf{p}, \frac{\mathbf{r}+\mathbf{r}^{\prime}}{2}\right)
$$

with the condensate wave function defined by Eq. (4.3). For the calculation of the thermal Wigner function we have to take into account that the low lying excited states of the interacting Bose gas are excitations of collective quasi-particle modes rather than single atoms. To be more specific, a proper treatment of the atomic interactions in the presence of a BoseEinstein condensate starts with decomposing the atomic field operator into a condensate and a fluctuation part 


$$
\hat{\Psi}(\mathbf{r})=\psi(\mathbf{r})+\delta \hat{\Psi}(\mathbf{r})
$$

with $\psi(\mathbf{r})$ obeying the Gross-Pitaevskii equation (4.1). Due to this choice of $\psi(\mathbf{r})$ only second order terms in the fluctuations $\delta \hat{\Psi}(\mathbf{r})$ and higher contribute to the interaction Hamiltonian. Within the Popov approximation third and fourth order terms in $\delta \hat{\Psi}(\mathbf{r})$ are treated in an averaged way, neglecting anomalous expectation values like $\langle\delta \hat{\Psi}(\mathbf{r}) \delta \hat{\Psi}(\mathbf{r})\rangle$. This extends the idea of the Bogoliubov approximation to finite temperatures since third and fourth order fluctuation terms are completely neglected in the latter. It is then possible to introduce quasi-particle operators $\hat{\alpha}_{j}$ which diagonalize the approximate Hamilton operator of the interacting system to

$$
\hat{H}=E_{0}+\sum_{j} \epsilon_{j} \hat{\alpha}_{j}^{\dagger} \hat{\alpha}_{j}
$$

The quasi-particle operators $\hat{\alpha}_{j}$ obey Bose commutation relations. In a spatially homogeneous system with volume $V$ they are related to the fluctuation operator $\delta \hat{\Psi}(\mathbf{r})$ by [33]

$$
\delta \hat{\Psi}(\mathbf{r})=\frac{1}{\sqrt{V}} \sum_{\mathbf{p}} e^{i \mathbf{p} \cdot \mathbf{r} / \hbar}\left(u_{\mathbf{p}} \hat{\alpha}_{\mathbf{p}}-v_{\mathbf{p}} \hat{\alpha}_{-\mathbf{p}}^{\dagger}\right) .
$$

By virtue of the local density approximation both the coefficients $u(\mathbf{p}, \mathbf{r})$ and $v(\mathbf{p}, \mathbf{r})$ as well as the Hartree-Fock energy

$$
\begin{aligned}
\epsilon_{\mathrm{HF}}(\mathbf{p}, \mathbf{r}) & =\frac{p^{2}}{2 m}+V(\mathbf{r})+2 U\left[|\psi(\mathbf{r})|^{2}+n_{T}(\mathbf{r})\right]-\mu \\
& =\frac{p^{2}}{2 m}+U|\psi(\mathbf{r})|^{2}
\end{aligned}
$$

and the Popov energy

$$
\begin{aligned}
\epsilon(\mathbf{p}, \mathbf{r}) & =\sqrt{\left[\epsilon_{\mathrm{HF}}(\mathbf{p}, \mathbf{r})\right]^{2}-\left[U|\psi(\mathbf{r})|^{2}\right]^{2}} \\
& =\sqrt{\left[\frac{p^{2}}{2 m}+U|\psi(\mathbf{r})|^{2}\right]^{2}-\left[U|\psi(\mathbf{r})|^{2}\right]^{2}}
\end{aligned}
$$

become functions of the position $\mathbf{r}$. The local spectrum of elementary excitations $\epsilon(\mathbf{p}, \mathbf{r})$ coincides with the well known Bogoliubov spectrum for a Bose condensed homogeneous gas close to zero temperature. The external potential and the interactions between uncondensed atoms influence the excitation spectrum only indirectly through the local condensate density (cf. Eq. (4.3)). If the kinetic energy of an elementary excitation with momentum $\mathbf{p}$ is smaller than the interaction energy of the condensate atoms, these excitations consist of phononlike quasi-particles. For large kinetic energies, the Popov energy $\epsilon(\mathbf{p}, \mathbf{r})$ coincides with the Hartree-Fock energy $\epsilon_{\mathrm{HF}}(\mathbf{p}, \mathbf{r})$ and the elementary excitations then consist of real particles instead.

Eq. (4.9) also explains why it was necessary to extend the local density approximation to the condensate wave function. Had we not dropped the kinetic energy of the condensate from the definition of the chemical potential in Eq. (4.1), the Popov energy would be given by 


$$
\epsilon(\mathbf{p}, \mathbf{r})=\sqrt{\left[\frac{p^{2}}{2 m}+U|\psi(\mathbf{r})|^{2}+\frac{\hbar^{2}}{2 m} \frac{\psi^{*}(\mathbf{r}) \nabla^{2} \psi(\mathbf{r})}{|\psi(\mathbf{r})|^{2}}\right]^{2}-\left[U|\psi(\mathbf{r})|^{2}\right]^{2}} .
$$

For a positive kinetic energy of the condensate wave function the argument of the square root could become negative, leaving the Popov energy undefined. One might try intuitively to remedy this problem, for example, by restricting the phase space available to the quasiparticles. However, numerical comparisons of several such schemes have yielded even less agreement with the results of an exact Path Integral Monte Carlo calculations [34 than the complete neglect of the quasi-particle excitations that corresponds to the Hartree-Fock method.

Finally, we obtain the thermal Wigner function by assuming the quasi-particle states to be occupied by a Bose-Einstein distribution and by expressing the mode functions $u(\mathbf{p}, \mathbf{r})$ and $v(\mathbf{p}, \mathbf{r})$ in terms of energies [30]

$$
\begin{aligned}
W_{T}(\mathbf{p}, \mathbf{r}) & =\frac{1}{(2 \pi \hbar)^{3}}\left[u^{2}(\mathbf{p}, \mathbf{r})+v^{2}(\mathbf{p}, \mathbf{r})\right] \frac{1}{\exp \left[\epsilon(\mathbf{p}, \mathbf{r}) / k_{B} T\right]-1} \\
& =\frac{1}{(2 \pi \hbar)^{3}} \frac{\epsilon_{\mathrm{HF}}(\mathbf{p}, \mathbf{r})}{\epsilon(\mathbf{p}, \mathbf{r})} \frac{1}{\exp \left[\epsilon(\mathbf{p}, \mathbf{r}) / k_{B} T\right]-1}
\end{aligned}
$$

Outside the condensate, collective effects do not play any role in determining the spectrum of elementary excitations. The first order correlation function is then given approximately by the formula (3.17) for an uncondensed ideal gas

$$
G^{(1)}\left(\mathbf{r}, \mathbf{r}^{\prime}\right)=\frac{1}{\lambda_{T}^{3}} g_{3 / 2}\left(\exp \left[\frac{\mu\left[\left(\mathbf{r}+\mathbf{r}^{\prime}\right) / 2\right]}{k_{B} T}\right], \exp \left[-\pi \frac{\left(\mathbf{r}-\mathbf{r}^{\prime}\right)^{2}}{\lambda_{T}^{2}}\right]\right),
$$

in which the local chemical potential $\mu(\mathbf{r})=\mu-V(\mathbf{r})-2 U n_{T}(\mathbf{r})$ is reduced by the meanfield interaction between the uncondensed atoms. However, these interactions are usually very weak and the correlation function is then essentially that discussed in Sec. [II].

A numerical plot of the volume integrated, normalized correlation function $g^{(1)}(\mathbf{r})$ for an interacting Bose condensed gas is given in Fig. 2. The effective strength of the atomic interaction is best expressed in terms of the universal scaling parameter $\eta=\mu_{T=0} / k_{B} T_{c}^{0}$ [30], where $T_{c}^{0}$ is the critical temperature of the ideal gas. This scaling parameter assumes values between 0.3 and 0.4 in most recent experiments. As in the case of a condensed ideal gas, the correlation function decreases for relative separations much larger than the thermal wavelength, but smaller than the total size of the condensate, to the overall condensate fraction, i.e., the system posesses an off-diagonal long range order. However, for an interacting gas, the tail of the correlation function decreases much more slowly, as a result of the presence of the long wavelength quasi-particle excitations. The unintegrated correlation function $G^{(1)}\left(\mathbf{r}, \mathbf{r}^{\prime}\right)$ depends strongly on its mean coordinate $\mathbf{R}=\left(\mathbf{r}+\mathbf{r}^{\prime}\right) / 2$ since the local condensate fraction varies significantly over the atomic sample.

\section{B. Second order correlation function}

Atomic interactions affect the second order correlation function in a more fundamental way than just by changing the spectrum of elementary excitations. Atoms repel each other 
strongly at small separations. It is therefore impossible to find two atoms at exactly the same location, i.e., $G^{(2)}(\mathbf{r}, \mathbf{r})=0$. This effect is clearly not accounted for by Eqs. (3.31) and (3.32). In order to study the influence of this hard core repulsion we briefly revisit the derivation of the time-dependent version of the Gross-Pitaevskii equation (4.1).

The condensate dyad $\psi(\mathbf{r}) \psi^{*}\left(\mathbf{r}^{\prime}\right)$ is part of the reduced one-particle density matrix $\rho^{(1)}$, which contains the same information as the first order correlation function. The dynamics of $\rho^{(1)}$ depends on the reduced two-particle density matrix $\rho^{(2)}$ through the Schrödinger equation

$$
\frac{\partial}{\partial t} \rho^{(1)}=-\frac{i}{\hbar}\left[H_{0,1}, \rho^{(1)}\right]-\frac{i}{\hbar} \operatorname{tr}_{2}\left\{\left[H_{I}, \rho^{(2)}\right]\right\}
$$

with the one-particle Hamiltonian $H_{0,1}$ and the true atomic interaction potential $U\left(\mathbf{r}_{1}-\mathbf{r}_{2}\right)$ in the coordinate representation

$$
H_{0, i}=-\frac{\hbar^{2} \nabla_{i}^{2}}{2 m}+V\left(\mathbf{r}_{i}\right), \quad H_{I}=U\left(\mathbf{r}_{1}-\mathbf{r}_{2}\right)
$$

The dynamics of the second order correlation function is similary linked to the third-order correlation function and so on, the entire set of $N$ coupled equations constituting the socalled BBGKY hierarchy. If it is possible, however, to express the reduced two-particle density matrix $\rho^{(2)}$ in terms of the reduced one-particle density matrix $\rho^{(1)}$, the hierarchy can be truncated by turning Eq. (4.13) into a closed nonlinear equation.

In most treatments, the time-dependent analog of the Gross-Pitaevskii equation (4.1) is obtained by replacing the true atomic interaction potential by the point interaction $\tilde{H}_{I}=U \delta\left(\mathbf{r}_{1}-\mathbf{r}_{2}\right)$ and by factorizing the reduced two-particle density matrix analogously to Eq. (3.31). Even though such an application of the first order Born approximation to a suitably chosen pseudopotential properly reproduces the Gross-Pitaevskii equation, its implications for quantities other than the reduced one-particle density matrix require more careful examination. For example, the above treatment implies that the second order correlation function

$$
G_{0}^{(2)}\left(\mathbf{r}, \mathbf{r}^{\prime}\right)=G^{(1)}(\mathbf{r}, \mathbf{r}) G^{(1)}\left(\mathbf{r}^{\prime}, \mathbf{r}^{\prime}\right)+\left|G^{(1)}\left(\mathbf{r}, \mathbf{r}^{\prime}\right)\right|^{2}-|\psi(\mathbf{r})|^{2}\left|\psi\left(\mathbf{r}^{\prime}\right)\right|^{2}
$$

corresponds to an ideal gas of quasi-particles with $G^{(1)}\left(\mathbf{r}, \mathbf{r}^{\prime}\right)$ given by Eq. (4.4). Such a conclusion, however, incorrectly ignores the strong repulsion between atoms at small distances.

We therefore outline a more detailed derivation of the Gross-Pitaevskii equation [23, 35] which also describes the two-particle density matrix appropriately. For this purpose we decompose the two-particle density matrix, $\rho^{(2)}=\rho_{0}^{(2)}+\delta \rho^{(2)}$, into a part $\rho_{0}^{(2)}$ representing products of the reduced single-particle density matrix (cf. Eq. (4.15)) and a part $\delta \rho^{(2)}$, which corrects for the true two-particle correlations. Rather than neglecting the correlation part $\delta \rho^{(2)}$ altogether, we turn Eq. (4.13) into a closed equation by solving the dynamics of $\delta \rho^{(2)}$ analytically and by reexpressing the correlations, $\delta \rho^{(2)}$, in terms of $\rho_{0}^{(2)}$. Of course, certain approximations have to be made in order to achieve a convenient analytical expression for $\delta \rho^{(2)}$.

The equation of motion of $\delta \rho^{(2)}$ is simplified by the assumption of an atom density low enough to permit neglect of three-body and higher order collisions. The two-particle 
correlations are therefore the result of two-particle scattering only. In the context of manyparticle physics this approach is often called a ladder approximation since the two-body collision is calculated up to arbitrary orders in the true interaction Hamiltonian $H_{I}$. It is worth noting that a literal point-like potential does not lead to any two-particle scattering, and that is consistent with the assumption $\rho^{(2)}=\rho_{0}^{(2)}$, but it is not at all realistic.

If we describe the two-particle scattering by the Møller operator

$$
\Omega=\lim _{t \rightarrow \infty} \exp \left[-i\left(H_{0,1}+H_{0,2}+H_{I}\right) t / \hbar\right] \exp \left[i\left(H_{0,1}+H_{0,2}\right) t / \hbar\right],
$$

it can be shown that the properly approximated two-particle density matrix is given by $\rho^{(2)}=\Omega \rho_{0}^{(2)} \Omega^{\dagger}$. Thus the equation of motion for $\rho^{(1)}$ reads [35]

$$
\frac{\partial}{\partial t} \rho^{(1)}=-\frac{i}{\hbar}\left[H_{0,1}, \rho^{(1)}\right]-\frac{i}{\hbar} \operatorname{tr}_{2}\left\{\left[H_{I}, \Omega \rho_{0}^{(2)} \Omega^{\dagger}\right]\right\} .
$$

The Møller operator $\Omega$ is related to the two-body $T$-matrix by the relation $T=H_{I} \Omega$. The matrix elements of the two-body $T$-matrix in momentum representation are identical to the scattering amplitude. It is therefore the $T$-matrix rather than the atomic interaction potential $H_{I}$ which should be approximated by a delta function $T=U \delta\left(\mathbf{r}_{1}-\mathbf{r}_{2}\right)$. To lowest order in the scattering length, this equation of motion can be further reduced to

$$
\frac{\partial}{\partial t} \rho^{(1)} \approx-\frac{i}{\hbar}\left[H_{0,1}, \rho^{(1)}\right]-\frac{i}{\hbar} \operatorname{tr}_{2}\left\{\left[T, \rho_{0}^{(2)}\right]\right\}
$$

which coincides with the previously outlined mean-field approximation. The neglected terms, which are quadratic in the scattering length, describe small thermal energy shifts and the dissipative effects of collisions such as thermalization [23].

We conclude from the above derivation of the Gross-Pitaevskii equation that the second order correlation function that properly accounts for hard core repulsion is given by

$$
\hat{G}^{(2)}=\Omega^{\dagger} \hat{G}_{0}^{(2)} \Omega .
$$

The correlation function is here defined as the operator $\hat{G}^{(2)}=\rho^{(2) \dagger}$ with the understanding that its coordinate representation corresponds to $G^{(2)}\left(\mathbf{r}, \mathbf{r}^{\prime}\right)$.

For reasons of simplicity we calculate $G^{(2)}$ first for an uncondensed gas. The scattering involves only the wave function for the relative coordinate $\mathbf{r}=\mathbf{r}_{1}-\mathbf{r}_{2}$ of the two atoms. If the incoming states are plane waves the effect of the Møller operator is given by [28]

$$
\Omega e^{i\left(\mathbf{p}_{1} \cdot \mathbf{r}_{1}+\mathbf{p}_{2} \cdot \mathbf{r}_{2}\right) / \hbar}=e^{i \mathbf{P} \cdot \mathbf{R} / \hbar}\left[e^{i \mathbf{p} \cdot \mathbf{r} / \hbar}-a \frac{e^{i p r / \hbar}}{r}\right] \quad(r \gg a)
$$

with the center-of-mass coordinate $\mathbf{R}=\left(\mathbf{r}_{\mathbf{1}}+\mathbf{r}_{2}\right) / 2$, the total momentum $\mathbf{P}=\mathbf{p}_{1}+\mathbf{p}_{2}$, and the relative momentum $\mathbf{p}=\left(\mathbf{p}_{1}-\mathbf{p}_{2}\right) / 2$. Here, we have assumed that all relative momenta are small enough to permit taking only the momentum independent part, $-a$, of the scattering amplitude into account. With this approximation we have neglected terms of order $p a / \hbar$ which is justified if $a \ll \lambda_{T}$. In a harmonic trap the ratio $a / \lambda_{T}$ is given by

$$
\frac{a}{\lambda_{T}}=\frac{4}{15} \frac{1}{\sqrt{\pi}} \frac{1}{\zeta(3)} \eta^{5 / 2}\left(\frac{T}{T_{c}}\right)^{1 / 2} .
$$


It is mainly determined by the scaling parameter $\eta=\mu_{T=0} / k_{B} T_{c}$ [30] which, as we have noted, assumes values between 0.3 and 0.4 in most recent experiments. As a consequence, we can generally assume a ratio of $a / \lambda_{T} \approx 0.01$ at the critical temperature.

In agreement with the local density approximation, we evaluate $G^{(2)}$ for a spatially homogeneous gas with volume $V$. Denoting the relative coordinate once more as $\mathbf{r}$ and the relative momentum as $\mathbf{p}$, we obtain for an uncondensed system (cf. Eq. (3.3))

$$
\begin{aligned}
G^{(2)}\left(\mathbf{r}_{1}, \mathbf{r}_{2}\right) & =\frac{1}{V^{2}} \sum_{\mathbf{p}_{1}, \mathbf{p}_{2}}\left(\Omega e^{i\left(\mathbf{p}_{1} \cdot \mathbf{r}_{1}+\mathbf{p}_{2} \cdot \mathbf{r}_{2}\right) / \hbar}\right)^{\dagger}\left(\Omega e^{i\left(\mathbf{p}_{1} \cdot \mathbf{r}_{1}+\mathbf{p}_{2} \cdot \mathbf{r}_{2}\right) / \hbar}\right)\left\langle n_{\mathbf{p}_{1}}\right\rangle\left\langle n_{\mathbf{p}_{2}}\right\rangle \\
& +\frac{1}{V^{2}} \sum_{\mathbf{p}_{1}, \mathbf{p}_{2}}\left(\Omega e^{i\left(\mathbf{p}_{1} \cdot \mathbf{r}_{1}+\mathbf{p}_{2} \cdot \mathbf{r}_{2}\right) / \hbar}\right)^{\dagger}\left(\Omega e^{i\left(\mathbf{p}_{2} \cdot \mathbf{r}_{1}+\mathbf{p}_{1} \cdot \mathbf{r}_{2}\right) / \hbar}\right)\left\langle n_{\mathbf{p}_{1}}\right\rangle\left\langle n_{\mathbf{p}_{2}}\right\rangle \\
& =\frac{1}{V^{2}} \sum_{\mathbf{p}_{1}, \mathbf{p}_{2}}\left(e^{i \mathbf{p} \cdot \mathbf{r} / \hbar}-a \frac{e^{i p r / \hbar}}{r}\right)^{\dagger}\left(e^{i \mathbf{p} \cdot \mathbf{r} / \hbar}-a \frac{e^{i p r / \hbar}}{r}\right)\left\langle n_{\mathbf{p}_{1}}\right\rangle\left\langle n_{\mathbf{p}_{2}}\right\rangle \\
& +\frac{1}{V^{2}} \sum_{\mathbf{p}_{1}, \mathbf{p}_{2}}\left(e^{i \mathbf{p} \cdot \mathbf{r} / \hbar}-a \frac{e^{i p r / \hbar}}{r}\right)^{\dagger}\left(e^{-i \mathbf{p} \cdot \mathbf{r} / \hbar}-a \frac{e^{i p r / \hbar}}{r}\right)\left\langle n_{\mathbf{p}_{1}}\right\rangle\left\langle n_{\mathbf{p}_{2}}\right\rangle .
\end{aligned}
$$

After multiplying this expression out term by term and making use of the spatial isotropy of the homogeneous gas we can write the second order correlation function as

$$
\begin{aligned}
G^{(2)}\left(\mathbf{r}, \mathbf{r}^{\prime}\right)= & G^{(1)}(\mathbf{r}, \mathbf{r}) G^{(1)}\left(\mathbf{r}^{\prime}, \mathbf{r}^{\prime}\right)\left(1+\frac{2 a^{2}}{\left|\mathbf{r}-\mathbf{r}^{\prime}\right|^{2}}\right)+\left|G^{(1)}\left(\mathbf{r}, \mathbf{r}^{\prime}\right)\right|^{2}\left(1-\frac{4 a}{\left|\mathbf{r}-\mathbf{r}^{\prime}\right|}\right) \\
= & {\left[G^{(1)}(\mathbf{r}, \mathbf{r}) G^{(1)}\left(\mathbf{r}^{\prime}, \mathbf{r}^{\prime}\right)+\left|G^{(1)}\left(\mathbf{r}, \mathbf{r}^{\prime}\right)\right|^{2}\right]\left(1-\frac{a}{\left|\mathbf{r}-\mathbf{r}^{\prime}\right|}\right)^{2} } \\
& +\left[G^{(1)}(\mathbf{r}, \mathbf{r}) G^{(1)}\left(\mathbf{r}^{\prime}, \mathbf{r}^{\prime}\right)-\left|G^{(1)}\left(\mathbf{r}, \mathbf{r}^{\prime}\right)\right|^{2}\right]\left[\left(1+\frac{a}{\left|\mathbf{r}-\mathbf{r}^{\prime}\right|}\right)^{2}-1\right]
\end{aligned}
$$

an expression which should also be valid for a sufficiently slowly varying inhomogeneous system. The first line of Eq. (4.23) multiplies the correlation function of an ideal gas by the squared relative wave function in the limit of zero momentum scattering. The additional term on the second line accounts for the fact that the atoms have finite relative momenta with a distribution implicitly characterized by $G^{(1)}\left(\mathbf{r}, \mathbf{r}^{\prime}\right)$. This correction term can only be neglected for very small $a / \lambda_{T}$ ratios. Furthermore, only linear terms in $a /\left|\mathbf{r}-\mathbf{r}^{\prime}\right|$ are relevant for distances $\left|\mathbf{r}-\mathbf{r}^{\prime}\right| \gg a$.

The extension of the previous calculation to Bose condensed gases is straightforward though it requires a little more algebra due to the existence of quasi-particle excitations. However, most of the required modifications are absorbed into the definition of the first order correlation function $G^{(1)}$. In addition one has to take into account the fact that the second order correlation function of a condensed ideal gas is given by Eq. (3.31) rather than by Eq. (3.3). With these modifications the second order correlation function of a condensed interacting gas is given by

$$
G^{(2)}\left(\mathbf{r}, \mathbf{r}^{\prime}\right)=G_{0}^{(2)}\left(\mathbf{r}, \mathbf{r}^{\prime}\right)\left(1-\frac{a}{\left|\mathbf{r}-\mathbf{r}^{\prime}\right|}\right)^{2}
$$




$$
\begin{array}{r}
+\left[G^{(1)}(\mathbf{r}, \mathbf{r}) G^{(1)}\left(\mathbf{r}^{\prime}, \mathbf{r}^{\prime}\right)-\left|G^{(1)}\left(\mathbf{r}, \mathbf{r}^{\prime}\right)\right|^{2}\right]\left[\left(1+\frac{a}{\left|\mathbf{r}-\mathbf{r}^{\prime}\right|}\right)^{2}-1\right] \\
g^{(2)}\left(\mathbf{r}, \mathbf{r}^{\prime}\right)=g_{0}^{(2)}\left(\mathbf{r}, \mathbf{r}^{\prime}\right)\left(1-\frac{a}{\left|\mathbf{r}-\mathbf{r}^{\prime}\right|}\right)^{2}+\left[1-\left|g^{(1)}\left(\mathbf{r}, \mathbf{r}^{\prime}\right)\right|^{2}\right]\left[\left(1+\frac{a}{\left|\mathbf{r}-\mathbf{r}^{\prime}\right|}\right)^{2}-1\right] .
\end{array}
$$

In Fig. 3 we compare the volume integrated normalized correlation functions $g^{(2)}$ and $g_{0}^{(2)}$ for an uncondensed system at the critical temperature of an ideal gas $T_{c}^{0}$. The interaction strength is given by the scaling parameter $\eta=0.31$. Even though the scattering length is much smaller than the thermal wavelength, with $a=0.007 \lambda_{T}$, the hard core repulsion significantly reduces the amount of bosonic attraction between the atoms. It becomes apparent from Fig. 4 that $g^{(2)}(\mathbf{r})$ is dominated by $g_{0}^{(2)}(\mathbf{r})(1-a / r)^{2}$ corresponding to the first line of Eq. (4.23). The additional terms contribute a $1 \%$ correction. They mainly suppress the residual effect of the hard core repulsion for distances of the order of the thermal wavelength and larger. Fig. Talso illustrates the diluting effect of the volume integration on the range of the bosonic attraction. For comparison, we plot in Fig. 5 the correlation function $g^{(2)}$ for a Bose condensed gas at $T=0.5 T_{0}$. Its maximum value is strongly reduced since condensate atoms do not exhibit any tendency to clump together due to wave function symmetrization. On the other hand, the existence of quasi-particle excitations leads to a relatively long tail for the correlation function. For this temperature, corrections to the term $g_{0}^{(2)}(\mathbf{r})(1-a / r)^{2}$ can be safely neglected, due to a larger thermal wavelength, i.e., $a / \lambda_{T}=0.005$, and the long tail of the correlation function.

\section{COHERENCE PROPERTIES}

We summarize in this section the coherence properties of trapped Bose gases based on our results for the first and second order correlation functions. First order coherence describes the ability of atoms with an initial separation $\mathbf{r}$ to form an interference pattern, when brought together. For an uncondensed gas, the visibility of such an interference pattern drops to zero on a length scale of $\mathbf{r}$ measured by the thermal wavelength, which is roughly of the order of $0.5 \mu \mathrm{m}$ in present experiments. In a Bose condensed gas, however, the visibility decreases on the same length scale to a plateau given by the total fraction of condensed atoms. This off-diagonal long range order allowed the experimental observation of an interference pattern [12] which extended over the whole cloud of condensate atoms. For spatial separations larger than the the size of the condensate, the visibility drops to zero as well, because long range order can not extend beyond the system size.

Second order coherence implies instead the absence of any density correlations other than the trivial autocorrelation pointed out in Eq. (2.8). In a dilute Bose gas, we can identify two sources of density correlations which limit the achievable degree of second order coherence. An inevitable anticorrelation between atoms originates from their strong mutual repulsion. The length scale of this repulsion is given by the scattering length $a$ which is of the order of a few nanometers and therefore much smaller than any other relevant length scale. However, the relative wave function of two atoms increases as $a / r$, which is to say very slowly. Anticorrelation effects are therefore still visible on the length scale of the thermal wavelength, which is about 200 times larger (at $T=0.5 T_{c}^{0}$ ) than the scattering 
length. The other limitation to second order coherence is due to the tendency of bosonic atoms to cluster together. The range of this effective bosonic attraction is again limited by the thermal wavelength. However, its maximal amount is a function of temperature too, since the condensate atoms do not exhibit such a clustering effect. Because of the interplay between hard core repulsion and bosonic clustering, the maximum of the second order correlation function depends on both the temperature and the scattering length.

It is nevertheless useful to characterize the local second order coherence by the maximum of the fictitious correlation function $g_{0}^{(2)}(\mathbf{r}, \mathbf{r})$ which does not account for hard core repulsion. We illustrate in Fig. 6 how this local measure of second order coherence, which is a function of the local condensate density, varies with the radial coordinate $r$ in a spherically symmetric system (cf. [18]). Analogously, the maximum $g_{0}^{(2)}(0)$ of the volume integrated correlation function offers a certain global measure of the second order coherence of the system. Fortunately, the quantity $g_{0}^{(2)}(0)$ is experimentally accessible due to its relation to the total atomic interaction energy. According to Eq. (4.18) the latter energy can be approximated by

$$
\left\langle H_{I}\right\rangle / 2=\operatorname{tr}\left\{H_{I} G^{(2)}\right\} / 2 \approx \operatorname{tr}\left\{T G_{0}^{(2)}\right\} / 2=\frac{U}{2} \int d \mathbf{r} G_{0}^{(2)}(\mathbf{r}, \mathbf{r})=\frac{U}{2} G_{0}^{(2)}(0) .
$$

The overlap integral between the true atomic interaction potential and the relative wave function between the atoms is therefore approximately proportional to the maximum value of the otherwise fictitious volume integrated correlation function $G_{0}^{(2)}(\mathbf{r})$.

In a recent experiment 15] the interaction energy and thereby $g_{0}^{(2)}(0)$ were determined by measuring the release energy of the atoms after switching off the magnetic trap. This experiment provided some evidence for a large degree of second order coherence of a trapped Bose-Einstein condensate at zero temperature. At finite temperature, however, a determination of the interaction energy from the release energy would require that the initial kinetic energy of the uncondensed atoms be measured independently. In a similar experiment the third order analog of $g_{0}^{(2)}(0)$ has been determined by measuring three-body scattering rates [16]. The implications of these experiments for second and third order coherence has been discussed before [15, 16, 18]. However, it was wrongly concluded that these experiments measure the true second order correlation function $G^{(2)}$ or its third order analog. The significant impact of the hard core repulsion on second and third order coherence has apparently not yet been fully appreciated.

Finally, we discuss the temperature dependence of $g_{0}^{(2)}(0)$ in Fig. 7 . The numerical plot shows a rapid but continuous decrease of $g_{0}^{(2)}(0)$ as the temperature falls below the critical temperature. A comparison with a calculation in the Hartree-Fock limit shows that the existence of quasi-particle excitations has a small but noticeable effect on second order coherence. We contrast these results with the second order coherence of both a spatially homogeneous and a harmonically trapped ideal Bose gas. In a spatially homogeneous medium one finds

$$
g^{(2)}(0)=2-\left(\frac{N_{0}}{N}\right)^{2}=2-\left[1-\left(\frac{T}{T_{c}}\right)^{3 / 2}\right]^{2},
$$

which is almost a linear function of temperature (cf. Fig. 7). In contrast the volume integrated second order correlation function of a harmonically trapped ideal Bose gas 


$$
\begin{aligned}
g^{(2)}(0) & =2-\frac{\int d \mathbf{r}|\psi(\mathbf{r})|^{4}}{\int d \mathbf{r} G^{(1)}(\mathbf{r}, \mathbf{r})^{2}}, \\
& =1+O(1 / \sqrt{N}), \quad T \leq T_{c}
\end{aligned}
$$

experiences a sharp transition from the value two above the critical temperature to the value one below it. The reason for this discontinuous transition is that the peak density of a harmonically trapped noninteracting Bose gas becomes infinite as the thermodynamic limit is reached while the peak density of the thermal cloud retains a finite value. Of course, the presence of atomic interactions prevents any such unbounded growth of the condensate density for a real gas. Nevertheless, the spatial inhomogeneity accelerates the transition to a second order coherent ensemble as the temperature is decreased.

\section{CONCLUSION}

We have extended the concept of optical coherence, defined in terms of correlation functions, to dilute trapped Bose gases. Except for a small region around the surface of a Bose-Einstein condensate, these correlation functions can be calculated by using a local density approximation. Even though the system behaves locally as if it were spatially homogeneous, the spatial dependence of the effective chemical potential adds some interesting new physical features. The influence of both the Bose-Einstein distribution of uncondensed atoms and of quasi-particle like excitations is strongly reduced when a volume average over the mean coordinate of the correlation functions is performed. Nevertheless, consequences of quasi-particle excitations are still noticeable in the tails of the correlation functions. The density correlation is dominated by a tendency of bosonic atoms to cluster together and, on the other hand, the existence of a hard core repulsion between these atoms. The quantum statistical attraction and the effect of coulomb repulsion are found to be of comparable range for the conditions of most recent experiments. It is in this sense incorrect to neglect the hard core repulsion in the discussion of density correlations.

For the discussion of second order coherence, however, it is convenient to introduce a fictitious correlation function which does omit the effect of hard core repulsion. This correlation function is experimentally accessible due to its close relation to the atomic interaction energy. By introducing this fictitious correlation function, we are able to explain the results of recent experiments on second and third order coherence. Finally, we have observed that a harmonically trapped gas achieves second order coherence much more rapidly as the temperature is lowered below the critical temperature than does its spatially homogeneous counterpart.

\section{ACKNOWLEDGMENTS}

It is a pleasure to thank M. Holzmann for stimulating discussions and the opportunity to compare our calculations with the unpublished results of a numerical Path Integral Monte Carlo calculation [36. M.N. acknowledges financial support by the Deutsche Forschungsgemeinschaft. 


\section{REFERENCES}

[1] O. Penrose and L. Onsager, Physical Review 104, 576 (1956).

[2] M. H. Anderson et al., Science 269, 198 (1995).

[3] C. C. Bradley, C. A. Sackett, J. J. Tollett, and R. G. Hulet, Physical Review Letters 75, 1687 (1995).

[4] K. B. Davis et al., Physical Review Letters 75, 3969 (1995).

[5] J. Javanainen and S. M. Yoo, Physical Review Letters 76, 161 (1996).

[6] M. Naraschewski et al., Physical Review A 54, 2185 (1996).

[7] W. Hoston and L. You, Physical Review A 53, 4254 (1996).

[8] J. I. Cirac, C. W. Gardiner, M. Naraschewski, and P. Zoller, Physical Review A 54, R3714 (1996).

[9] T. Wong, M. J. Collett, and D. F. Walls, Physical Review A 54, R3718 (1996).

[10] H. Wallis, A. Röhrl, M. Naraschewski, and A. Schenzle, Physical Review A 55, 2109 (1997).

[11] Y. Castin and J. Dalibard, Physical Review A 55, 4330 (1997).

[12] M. R. Andrews et al., Science 275, 637 (1997).

[13] A. Röhrl, M. Naraschewski, A. Schenzle, and H. Wallis, Physical Review Letters 78, 4143 (1997).

[14] H. Wallis et al., Journal of Modern Optics 44, 1751 (1997).

[15] W. Ketterle and H.-J. Miesner, Physical Review A 56, 3291 (1997).

[16] E. A. Burt et al., Physical Review Letters 79, 337 (1997).

[17] R. J. Glauber, Physical Review 130, 2529 (1963).

[18] R. J. Dodd, C. W. Clark, M. Edwards, and K. Burnett, Optics Express 1, 284 (1997).

[19] F. Sols, Physica B 194-196, 1389 (1994).

[20] E. M. Wright, D. F. Walls, and J. C. Garrison, Physical Review Letters 77, 2158 (1996).

[21] M. Lewenstein and L. You, Physical Review Letters 77, 3489 (1996).

[22] M. Naraschewski, A. Röhrl, H. Wallis, and A. Schenzle, Materials Science \& Engineering B48, 1 (1997).

[23] M. Naraschewski, Dissertation, Universität München, 1997.

[24] A. Imamoḡlu, M. Lewenstein, and L. You, Physical Review Letters 78, 2511 (1997).

[25] D. Jaksch, C. W. Gardiner, K. M. Gheri, and P. Zoller, cond-mat/9712206 (unpublished).

[26] U. M. Titulaer and R. J. Glauber, Physical Review 140, B676 (1965).

[27] E. Hecht, Optics, 3 ed. (Addison-Wesley, Reading, 1998).

[28] J. J. Sakurai, Modern quantum mechanics (Addison-Wesley, Reading, 1994).

[29] V. Bagnato, D. E. Pritchard, and D. Kleppner, Physical Review A 35, 4354 (1987).

[30] S. Giorgini, L. P. Pitaevskii, and S. Stringari, Journal of Low Temperature Physics 109, 309 (1997).

[31] H. D. Politzer, Physical Review A 54, 5048 (1996).

[32] V. V. Goldman, I. F. Silvera, and A. J. Leggett, Physical Review B 24, 2870 (1981).

[33] A. L. Fetter and J. D. Walecka, Quantum Theory of Many-Particle Systems (Mc GrawHill, New York, 1971).

[34] M. Holzmann, W. Krauth, and M. Naraschewski, unpublished.

[35] R. Balescu, Equilibrium and Nonequilibrium Statistical Mechanics (John Wiley \& Sons, New York, 1975). 
[36] M. Holzmann and Y. Castin, unpublished. 


\section{FIGURES}

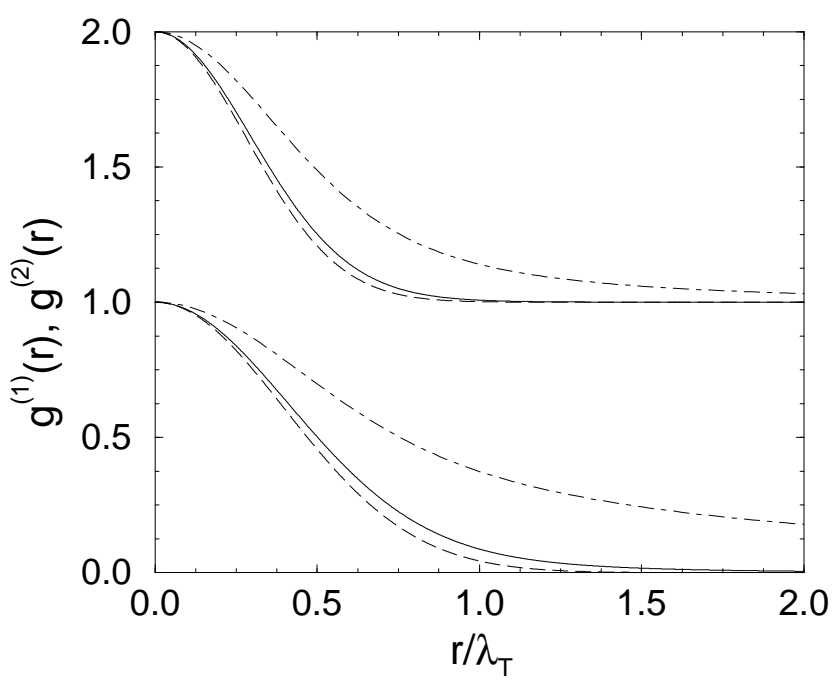

FIG. 1. This plot shows the volume integrated first order (bottom) and second order (top) correlation functions for a harmonically trapped ideal gas at the critical temperature. The solid lines correspond to the exact expressions of Eqs. (3.22) and (3.4), while the dashed lines approximate the occupation distribution of the trap levels by a Maxwell-Boltzmann distribution as in Eq. (3.23). In order to display the diluting effect of the volume integration, implicit in these results, we have added as dot-dashed lines the unintegrated correlation functions for mean positions at the center of the trap, where the local chemical potential is the smallest.

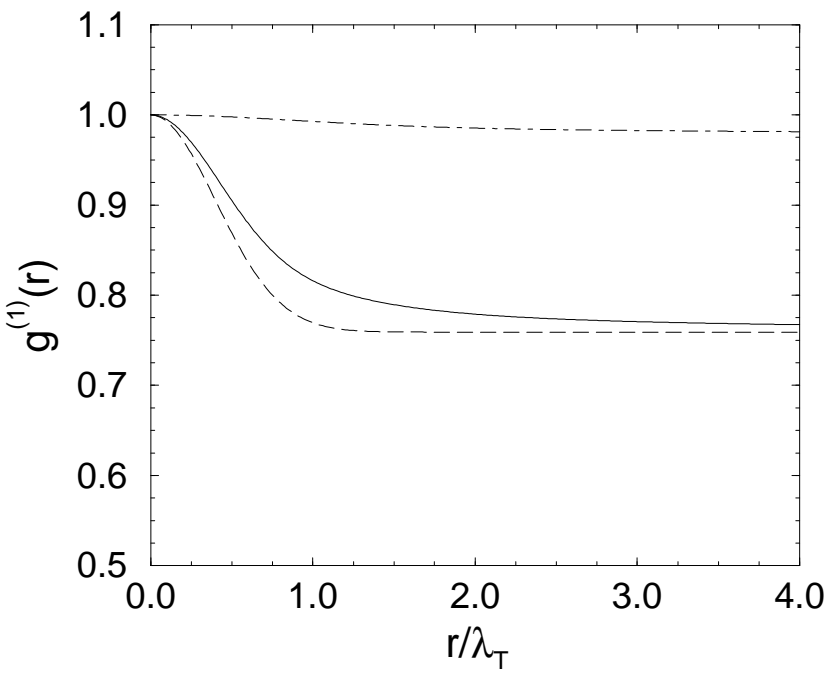

FIG. 2. The solid line shows the volume integrated first order correlation function for a harmonically trapped interacting gas at $T=0.5 T_{c}^{0}$ where $T_{c}^{0}$ is the critical temperature of the ideal gas. The scaling parameter is $\eta=0.31$. The correlation function does not decay to zero due to the existence of a Bose-Einstein condensate. As in Fig. 1, the dashed line corresponds to a Maxwell-Boltzmann distribution of the uncondensed atoms while the dot-dashed line gives the unintegrated correlation function at the center of the trap. The existence of quasi-particle excitations leads to a significantly slower decay of the tail of the correlation function, compared to the case of an ideal gas.

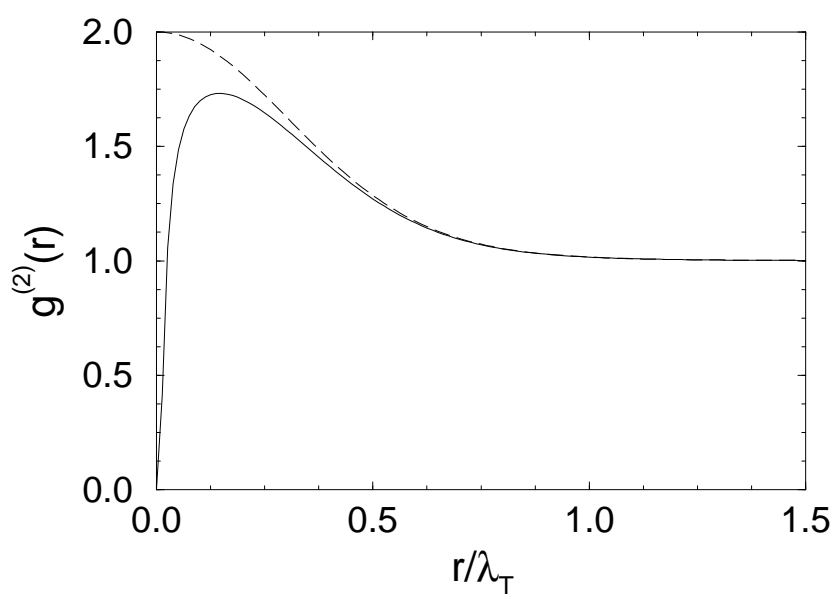


FIG. 3. The volume integrated second order correlation function of an interacting gas is given by the solid line. The temperature corresponds to the critical temperature $T_{c}^{0}$ of an ideal gas. With a scaling parameter of $\eta=0.31$, the scattering length $a$ is much smaller than the thermal wavelength, $a=0.007 \lambda_{T}$. Nevertheless, the hard core repulsion leads to noticeable reduction of the correlation function over a broad range of relative distances. The dashed line shows as a comparison the volume integrated fictitious correlation function $g_{0}^{(2)}(\mathbf{r})$ which omits the effect of the hard core repulsion.

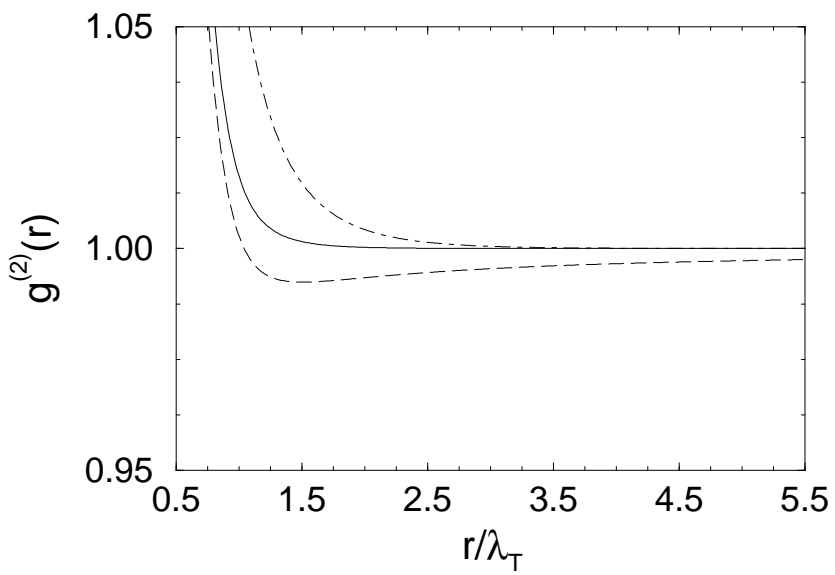

FIG. 4. This plot compares the long range behavior of the volume integrated second order correlation function of Fig. 3 (solid line) with its main constituent $g_{0}^{(2)}(r)(1-a / r)^{2}$ (dashed line). The latter approximates the relative wave function by its zero temperature limit. The difference is small, and becomes even smaller at lower temperatures. The dot-dashed line, by contrast, displays the unintegrated correlation function at the center of the trap.

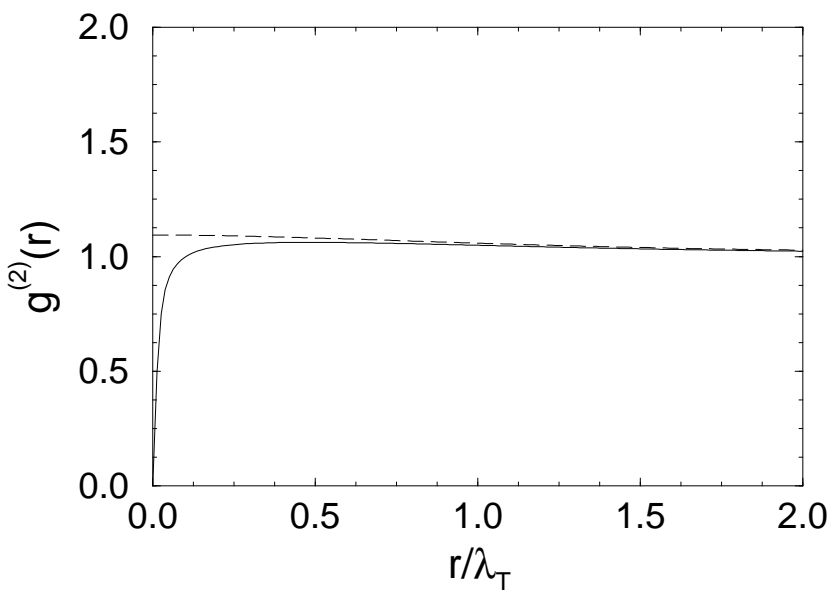

FIG. 5. The volume integrated second order correlation function is here plotted for a temperature $T=0.5 T_{c}^{0}$. The dashed line shows $g_{0}^{(2)}(\mathbf{r})$ which omits the hard core repulsion. Due to the presence of a considerable condensate fraction, a large degree of second order coherence is attained. The existence of quasi-particle excitations leads to a remarkably slow decrease of the correlation function.

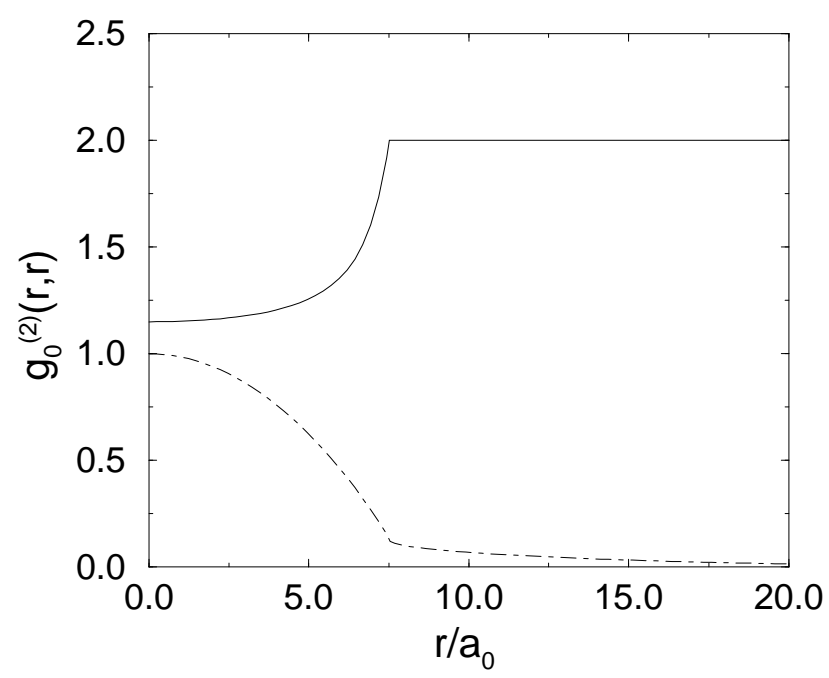


FIG. 6. The normalized second order correlation function $g_{0}^{(2)}(\mathbf{r}, \mathbf{r})$ of Eq. 3.34) is a measure of local second order coherence. It also describes the ratio of the total interaction energy, including quantum statistical exchange energy, to the classical Hartree mean-field energy. We have plotted $g_{0}^{(2)}(\mathbf{r}, \mathbf{r})$ (solid line) for a harmonically trapped gas with interaction strength $\eta=0.31$ and temperature $T=0.8 T_{c}^{0}$. For comparison, the dot-dashed curve shows the total density distribution in arbitrary units.

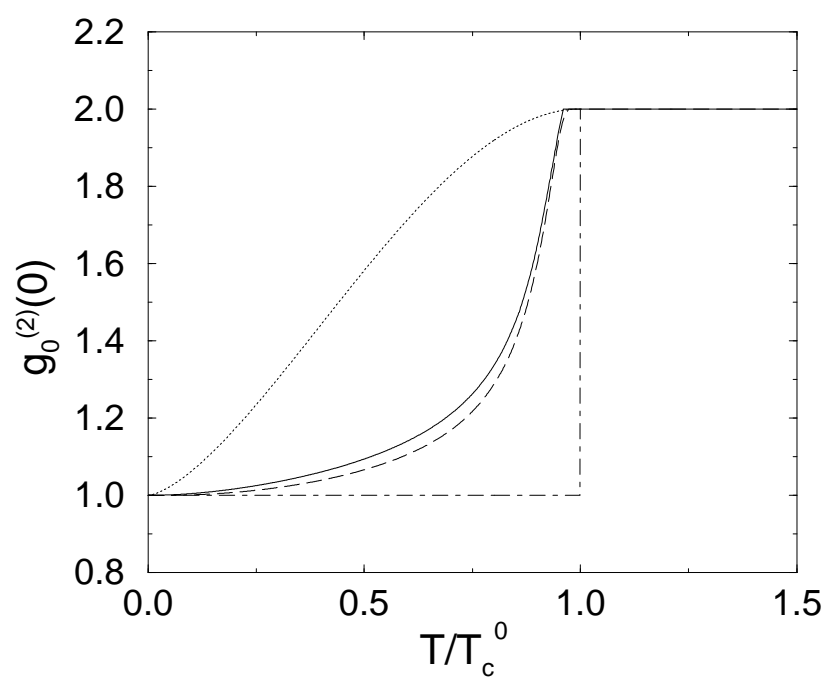

FIG. 7. The peak value $g_{0}^{(2)}(0)$ of the volume integrated second order correlation function, excluding the hard core repulsion between the atoms, is a good measure of the second order coherence of the system. The solid line shows the influence of the temperature on the second order coherence for a harmonically trapped interacting gas in the Popov approximation with scaling parameter $\eta=0.31$. A corresponding calculation using the Hartree-Fock approximation (dashed line) shows that quasi-particle excitations have a small but noticeable effect. For comparison, the dotted line describes a spatially homogeneous ideal gas while the dot-dashed line corresponds to a harmonically trapped ideal gas. 